\title{
Classification and Detection of Citrus Disease using Feature Extraction and Support Vector Machine (SVM)
}

\author{
Ayobami I. Ojelabi \\ Computer Science Dept., \\ University of Ibadan \\ Nigeria
}

\author{
Oluwabusayo I. Omotosho \\ Computer science Department \\ University of Ibadan \\ Nigeria
}

\author{
Olajide A. Oladejo \\ Computer Science Dept., \\ University of Ibadan \\ Nigeria
}

\begin{abstract}
In agriculture, plant infections are responsible for the reduction in the production of citrus fruits which causes a major economic loss. In plants, citrus is used as a major source of nutrients like vitamin $\mathrm{C}$ throughout the universe. However, 'Citrus' diseases badly affect the production and quality of citrus fruits. Over the years, image processing techniques have been widely used for detection and classification of citrus diseases but a novel model for such challenge has not been properly explored. Hence, the need for a model that will be able to label the diseases accordingly.
\end{abstract}

A model for detection and classification of citrus diseases using feature selection and support vector machine (SVM) was developed. The method consists of two primary phases; (a) detection of lesion spots on the citrus fruits; (b) classification of citrus diseases. The citrus lesion spots are extracted by an optimized segmentation method using Kmeans, which was performed on an enhanced citrus image. The selected features are fed into Support Vector Machine (SVM) for the citrus disease classification, and the model tested with various test images that consist of healthy and diseased citrus fruits.

The model shows a better performance than previous models at $95 \%$ accuracy.

\section{General Terms}

Machine Learning, Agriculture

\section{Keywords}

Support Vector Machine, Feature Extraction, Feature

Selection, Citrus Disease

\section{INTRODUCTION}

Fruit plants are a major part of any agro economical society [17]. Among fruit plants, citrus plants are packed with Vitamin-C, which provide multiple benefits to human health and are also used as a raw material in several agro-industries [20]. However, in recent years, citrus production is widely affected by citrus diseases. These citrus diseases include canker, greasy spot, and black spot. In this regard, several automated solutions have been proposed for the symptombased detection of citrus plant diseases, and promising results were recorded. Therefore, in present times, researchers are striving to find novel computer-based solutions for the early identification of citrus diseases, from the field of image processing [19].

Biochemical techniques that very sensitive have been developed in recent years, which allow diagnosis within hours. However, they are highly expensive and require instrumental and specific training. Thus, collaborative efforts are required to improve the current procedure of visual diagnosis for disease to be detected earlier [4], such as technological strategies using machine learning to achieve intelligent farming [7]. Recent studies examine the problem of post-harvest processing of citrus (Gomez-Sanchez et al., 2012), most especially, visual detection defects through image analysis to classify the fruit depending on appearance in an unsupervised way [5] [4].

Agriculture is now conceived as been much more than just feeding the ever-growing population of the world. Agriculture is the backbone of our economy as it not only provides food but raw materials for industry. Plants are not only the source food but also important as a source of energy-rich compounds, vitamins, minerals, specifically the importance of citrus in the agriculture and world is exhibited by its large-scale production and wide-reaching provision. It is referred to as the key genus to the family of Rutaceae for agriculture. Numerous kinds of citrus are well- thought-out to be inherent to both tropical and sub-tropical areas of Asia and Malaya archipelago. It positions carefully potential value and is elevated in more than 52 countries of the world [24].

Citrus plants are prone to diseases such as Citrus canker [22], Gummosis, Citrus greening (Gre) [23], anthracnose (Ant) [25], and downy (Dow) [26]. To control these diseases, a large number of chemicals or fungicides are used on the citrus crop, which results in both economic loss and environmental pollution. In this context, proper diseases diagnosis in a timely and accurate manner is of uttermost importance. If these diseases are not properly diagnosed and controlled, it will adversely affect citrus production in coming years. The management of recurrent crops requires close monitoring to tackle diseases that can affect crop production significantly and successively after harvest life. The major citrus diseases can be observed and classified by experts on the basis of their symptoms. But this requires continuous monitoring and manual observation which could be error-prone and costly.

The image processing technology is starting to play a crucial role in several agricultural applications such as weed control, crop fertilization, plant species recognition and detection, growing phase determination, plant disease detection, and harvesting fruits. In general, computer vision is used to detect specific plants in a sequence of video, based on a combination of feature extraction and classification. Effective feature extraction and region detection in an image can help to reduce the superfluous key points and improve the efficiency of the system (computational time).

Moreover, [29] reported that the real challenge for feature detection and posterior image matching is to achieve robust feature detection with the following characteristics: (i) consistency: positions of objects detected should be insensitive to noise, scale, orientation, clutter, illumination; (ii) accuracy: objects should be detected as close as possible to the correct positions and features; (iii) speed: should be sufficiently fast. Also, challenging real-world conditions like 
weather variability, presence of shadows in sunny conditions, natural similarities between the target object (weed or crop) and the background, impair the performance of machine vision techniques [2].

One of the major challenges that affects the plant-based segmentation accuracy mostly is sunny conditions as it leads to similarities in color between crops and weeds. For example, when the sunshine is strong, the surface of some leaf types (such as cauliflower or corn leaf), acts like a mirror (spectacular reflection); as a result, it may be segmented into the wrong category [2]. Dealing with a moving camera may generate issues such as vibration, speed variations, and crops inadvertently covered by soil caused by moving machinery.

These problems translate into a loss of detection of moving objects between frames and may result in the e.g. inaccurate operation of automatic weeding or spraying machinery that relies on accurate detection of a plant across multiple frames. This paper addresses the problem of reducing detection errors in sunny conditions in particular, through the application of multiple-object tracking and data association. Therefore, in underdeveloped countries, where most farmers are uneducated, have to pay for such cost in addition to other expenses (e.g. fertilizer and pesticides). Moreover, farmers are unaware of non-native diseases [21].

However, 'Citrus' diseases badly affect the production and quality of citrus fruits. Over the years, the computer vision and image processing techniques have been widely used for detection and classification of diseases in plants. In this article, I propose a hybrid method for detection and classification of diseases in citrus plants. The proposed method consists of two primary phases; (a) detection of lesion spots on the citrus fruits and leaves; (b) classification of citrus diseases. The citrus lesion spots are extracted using an optimized weighted segmentation method, which is performed on an enhanced input image. Then, color, texture, and geometric features are fused and saved in a codebook.

\section{RELATED WORKS}

A very early attempt to monitor plant health was carried out by [15]. The model tried to identify deficiencies of iron, zinc and nitrogen by monitoring lettuce leaves. The capture of the images was done by an analog video camera, and only afterward the images would be digitized. the segmentation of the images into leaf and background was the first step of the proposed algorithm.

[6] compared two different approaches to detect and classify three types of citrus diseases. The authors collected 39 texture features samples and made four different subsets of the features to be used in two different classification approaches. The first approach used Mahalanobis minimum distance classifier, using the nearest neighbor principle, while radial basis functions (RBF) neural network classifiers trained with the backpropagation algorithm was the second approach. The two classification approaches performed equally well when using the best of the four subsets, which contained ten hue and saturation texture features.

[13] proposed a method to detect and classify three different types of diseases that affect Phalaenopsis orchid seedlings. The segmentation procedure adopted by the author is significantly more sophisticated than those found in other papers, which composed of four steps: removal of the plant vessel using a Bayes classifier, equalization of the image using an exponential transform, a rough estimation for the location of the diseased region, and equalization of the subimage centered at that rough location. A number of color and texture features are then extracted from the gray level cooccurrence matrix [14].

Finally, those features are fed into an MLP artificial neural network with one hidden layer for final classification. [9] tackled the problem of detecting and classifying six types of mineral deficiencies in rice crops. Firstly, the algorithm was made to extract a number of texture and color features with each feature (texture and color) submitted to its own specific MLP neural network. The two networks have one hidden layer, but different number of neurons in the hidden layer (40 for texture and 70 for color). The results of these networks are then combined, yielding the final classification. The author used a similar approach in [8], but in this case, the objective was to identify two kinds of diseases (blast and brown spots) that affect rice crops.

[18] proposed a method to identify and classify diseases that affect grapevines. The approach uses many color representations (HSI, L*a*b*, UVL, and $\mathrm{YCbCr}$ ) throughout its execution. The separation between leaves and background is done by an MLP neural network, which is coupled with a color library built a priori by means of an unsupervised selforganizing map (SOM). The colors present on the leaves are then clustered by means of an unsupervised and untrained self-organizing map. the number of clusters to be adopted in each case was determined by a genetic algorithm. Support Vector Machine (SVM) are then used to determine the Diseased and healthy regions. After some additional manipulations, the segmented image is submitted to a multiclass SVM, which performs the classification into a scab, rust, or no disease.

[12] proposed a method to identify two diseases that can manifest in cucumber leaves. statistic pattern recognition technique was used to achieve the segmentation into healthy and diseased regions. color, shape and texture features are extracted and fed into the SVM, for final classification. It was recorded SVM performed far better than neural networks in terms of results provided.

[16] used SVMs to classify feature points descriptors generated using Harris corner points, SURF descriptors and SIFT descriptors for pineapple fruit detection, counting, and tracking. The color, shape and texture features of pineapple were extracted from 100 images. The classification was then carried out with the Radial Basis Function (RBF) SVM kernel function based on color, and shape as well as integrated colorshape features. The fruit detection accuracy was $91.3 \%$ when the RBF kernel function was implemented based on integrated color and shape features. The authors reported a false positive of $0.39 \%$ per frame.

The system proposed by Yao et al. [10] aimed to identify and classify three types of diseases that affect rice crops. The algorithm first applies a particular color transformation to the original RGB image, resulting in two channels ( $y 1$ and $y 2)$. The image is then segmented using Otsu's method, after which the diseased regions are isolated, and the extracted Color, shape and texture features are then fed to a Support Vector Machine for final classification.

[11] proposed a model to recognize three kinds of cucumber leaf diseases. Similar to most approaches, a variety of color, shape and texture features are extracted by a simple thresholding procedure to the separate between healthy and diseased regions. These features are then fed to an SVM with Radial Basis Function (RBF) as a kernel, which performs the final classification. 


\section{METHODOLOGY}

For the demonstration of the proposed system, dataset of citrus images was created with the help of a domain expert. Images captured with a digital camera and some images were acquired from the internet because of the unavailability of canker and greasy spot diseases in our region at the present time. There are 200 samples of citrus images used as samples in the testing and training stage of this research work. The proposed method is a conjunction of four primary steps consisting of: Image enhancement, identification of infected region using lesion segmentation, feature extraction and construction of codebook, feature selection and classification.

The following algorithm summarizes the processing performed by the new system

1) Acquire input Image $I$.

2) Perform Image pre-processing

3) Detect fruit diseased regions in the first image of I. Let $\mathrm{Ni}$ be the number of detected fruit regions and let $R_{i}, I \in\{1 \ldots$, No $\}$, be the set of pixels in region $i$.

4) For each subsequent image is of I,

a) Detect fruit regions $\left\{R_{i}\right\} I, I \in 1 \ldots, N_{j}$ in $I_{j}$.

b) Classify the healthy and diseased citrus fruit $\left\{R_{i}\right\}$ $\mathrm{i}, \mathrm{i} \in 1, \ldots . . \mathrm{N}$ into new fruit regions not associated with existing trajectories and existing fruit regions associated with existing trajectories based on whether or not they overlap with a fruit detection in the previous image.

c) For each fruit region $R_{i}$ overlapping with one or more fruit regions in image $\mathrm{j}-1$, find $\mathrm{k}$ such that $\mathrm{R} \kappa$ $\mathrm{j}-1$ is the largest of such region, then add region $R \kappa, j$ $=\mathrm{R}_{\mathrm{i}}$ to $\mathrm{Tk}$

5. Perform morphological processing of the identified fruit regions.

6. Perform the classification of the identified fruits using SVM classifier

7. Each of the identified fruit regions will be represented by a subplot, each subplot is a number of diseased and healthy citrus fruits and the total will be the result of our classification of various citrus disease.

\subsection{Lesion segmentation}

Image segmentation is an important step in pattern recognition and image processing-based applications. In plants, the lesion segmentation step is used for the detection of lesion spot in the infected images automatically. To handle the automatic detection of lesion spots in the images, a weighted segmentation technique based on the Chi-square distance and threshold function is implemented. Moreover, the weighted segmentation method is optimized with existing High Dimensional Color Transform (HDCT) based saliency (Kim, 2016) technique to obtain a new segmented image, which has better results as compared to saliency segmentation method.
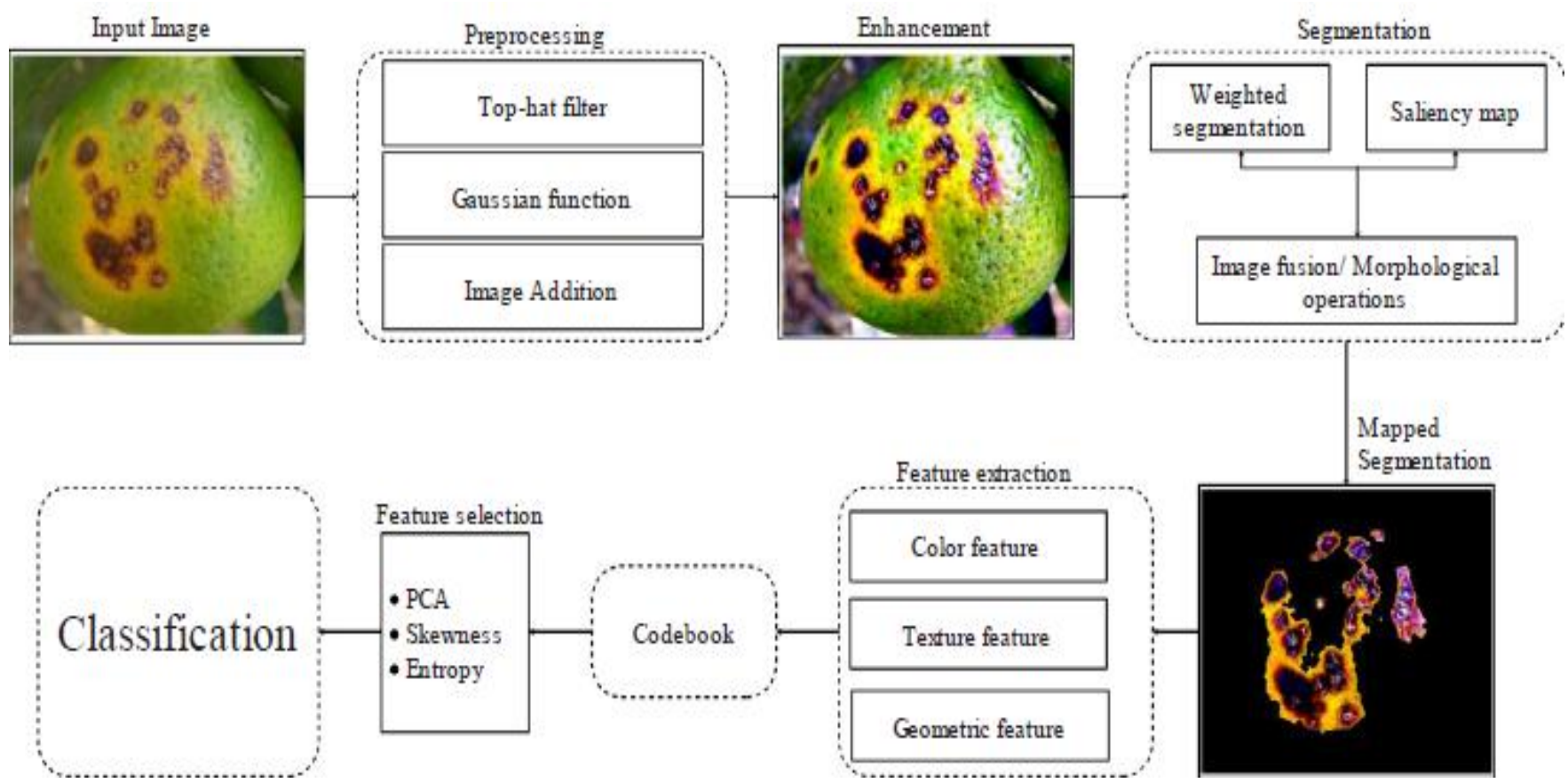

Fig.1: System architecture for identification and classification of infected plants diseases.

\subsection{Feature extraction}

Feature extraction plays a vital role in the field of image processing and pattern recognition for the representation of an image. In this section, the major issue is efficient feature extraction and selection for accurate classification of infected regions.
To solve this problem, a codebook was constructed by extraction of three types of features including texture, color, and geometric. The extracted features are stored into a codebook and best features for classification were selected based on their maximum score achieved after application of our proposed feature selection approach. The flow diagram of feature extraction is shown in Fig 2. 


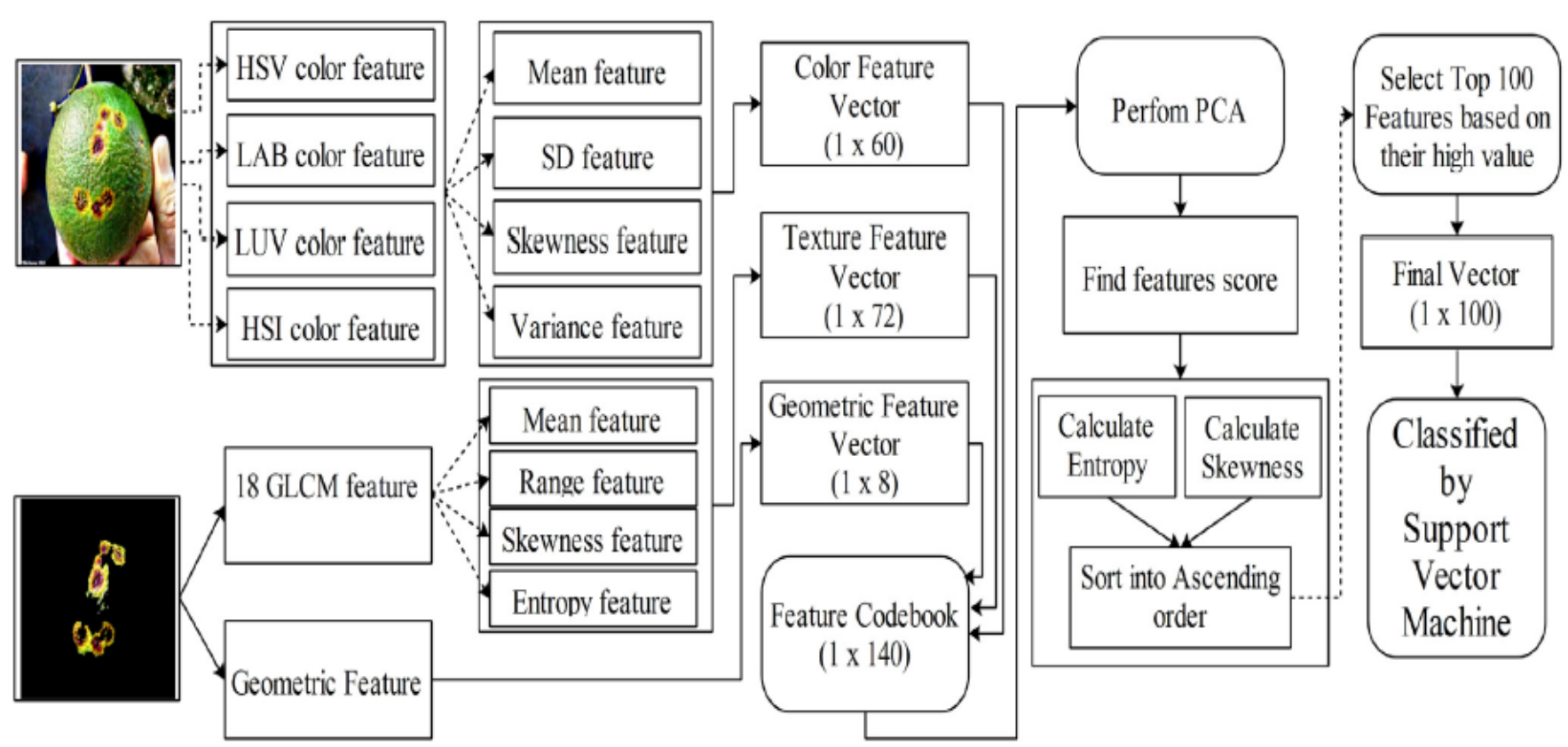

Fig.2: Flow diagram for Codebook construction and then feature selection.

\subsubsection{Color feature}

The color features are extracted from enhanced images, which are obtained from the section above. The color features are more important for the classification of the infected region in the image because each lesion spot has a different color. These features are obtained by the use of different types of color spaces and parameters. In this work, five types of color spaces were used for color features: Enhanced RGB, HSV, HSI, LAB, and LUV.

\subsubsection{Texture feature}

The purpose of texture feature extraction is to observe the combination of image intensities at the distinct positions, measure the level of irregularity, midpoint, smallest interval between diseased pixels, and shape of disease spots, which are relative to each other in the image. The numbers of pixels in the image are classified into first order, second order, and third order. Four new features such as cluster prominence and shade, homogeneity, and energy are added in to achieve best classification accuracy. The cluster shade and cluster prominence features can be modified by a sum histogram problem and homogeneity feature can be calculated from difference histogram problems.

\subsection{Codebook Construction}

The codebook was constructed after extraction of three types of features including color, geometric and texture. These features are constructed into a codebook by simple concatenation based fusion method. The feature fusion means to fuse multiple features or description of multiple features in one vector, which contains more information as compared to the individual feature vector. In literature, several types of feature fusion techniques have been implemented such as discriminant correlation analysis [28] and CCA [27].
However, due to paucity of a large number of features, simple concatenation-based feature fusion was used. The utilized method maximizes the correlation across the extracted set of features and omits between class variations at the same time. Hence, the size of the feature codebook is $1 \times 140$, which is later optimized by a new feature selection method. In the feature selection method, the best features were selected for final classification.

\subsection{Feature selection}

In this work, a hybrid feature selection technique was implemented based on PCA score, Entropy, and Covariance, for the following reasons;

(i) To improve the classification accuracy by solving the problem of overfitting;

(ii) To improve the prediction performance for a supervised method such as SVM etc., and

(iii) To remove the redundancy between features.

The approach used in this research work, initially, the fused codebook features are fed to PCA as input to find out their score value. Then entropy and covariance are performed on score vector separately and sorted into ascending order. Thereafter, the top 100 features were selected from each and fused in parallel based on the maximum value.

\section{RESULTS AND EVALUATION}

In this section, the results from the study are discussed. Table 1 shows the fruit color extraction which consist of parameters, such as Entropy, variance and skewness with their corresponding values for each citrus disease dataset sample, these are canker, anthracnose, and black spot respectively. 
Table 1: Fruit color extraction

\begin{tabular}{|c|c|c|c|}
\hline Parameters & Entropy & Variance & Skewness \\
\hline \multirow{5}{*}{$\begin{array}{l}\text { Citrus fruits affected with } \\
\text { canker }\end{array}$} & 0.1288 & 3.154 & 0.007 \\
\hline & 0.1200 & 3.526 & 0.010 \\
\hline & 0.1276 & 3.176 & 0.015 \\
\hline & 0.1267 & 3.290 & 0.019 \\
\hline & 0.1200 & 3.145 & 0.039 \\
\hline \multirow{5}{*}{$\begin{array}{c}\text { Citrus fruits affected with } \\
\text { anthracnose }\end{array}$} & 0.0990 & 4.764 & 0.008 \\
\hline & 0.0010 & 4.797 & 0.011 \\
\hline & 0.0010 & 4.753 & 0.005 \\
\hline & 0.0900 & 3.876 & 0.015 \\
\hline & 0.0767 & 4.296 & 0.005 \\
\hline \multirow{5}{*}{$\begin{array}{c}\text { Citrus fruits affected with } \\
\text { Black spot }\end{array}$} & 0.0300 & 5.266 & 0.095 \\
\hline & 0.0091 & 5.465 & 0.065 \\
\hline & 0.0091 & 5.368 & 0.069 \\
\hline & 0.0092 & 4.945 & 0.056 \\
\hline & 0.009 & 5.234 & 0.093 \\
\hline
\end{tabular}

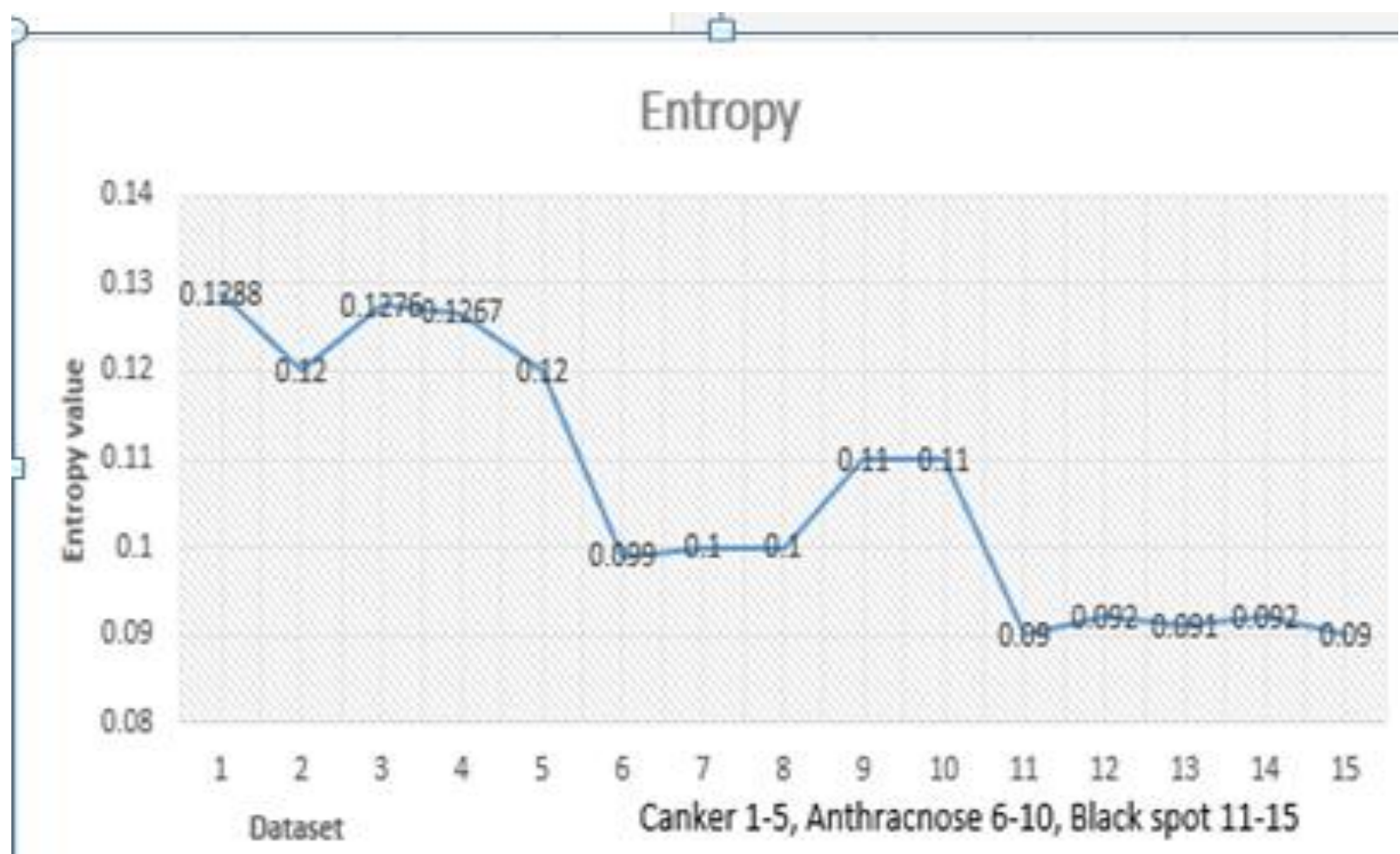

Figure 3. Citrus fruit Entropy result graph

Figure 3, shows entropy values plotted against various citrus infections namely canker, anthracnose, and black spot. It is observed that citrus canker disease has the highest curve which implies that our method was able to detect and classify canker disease after computation. 


\section{Variance}

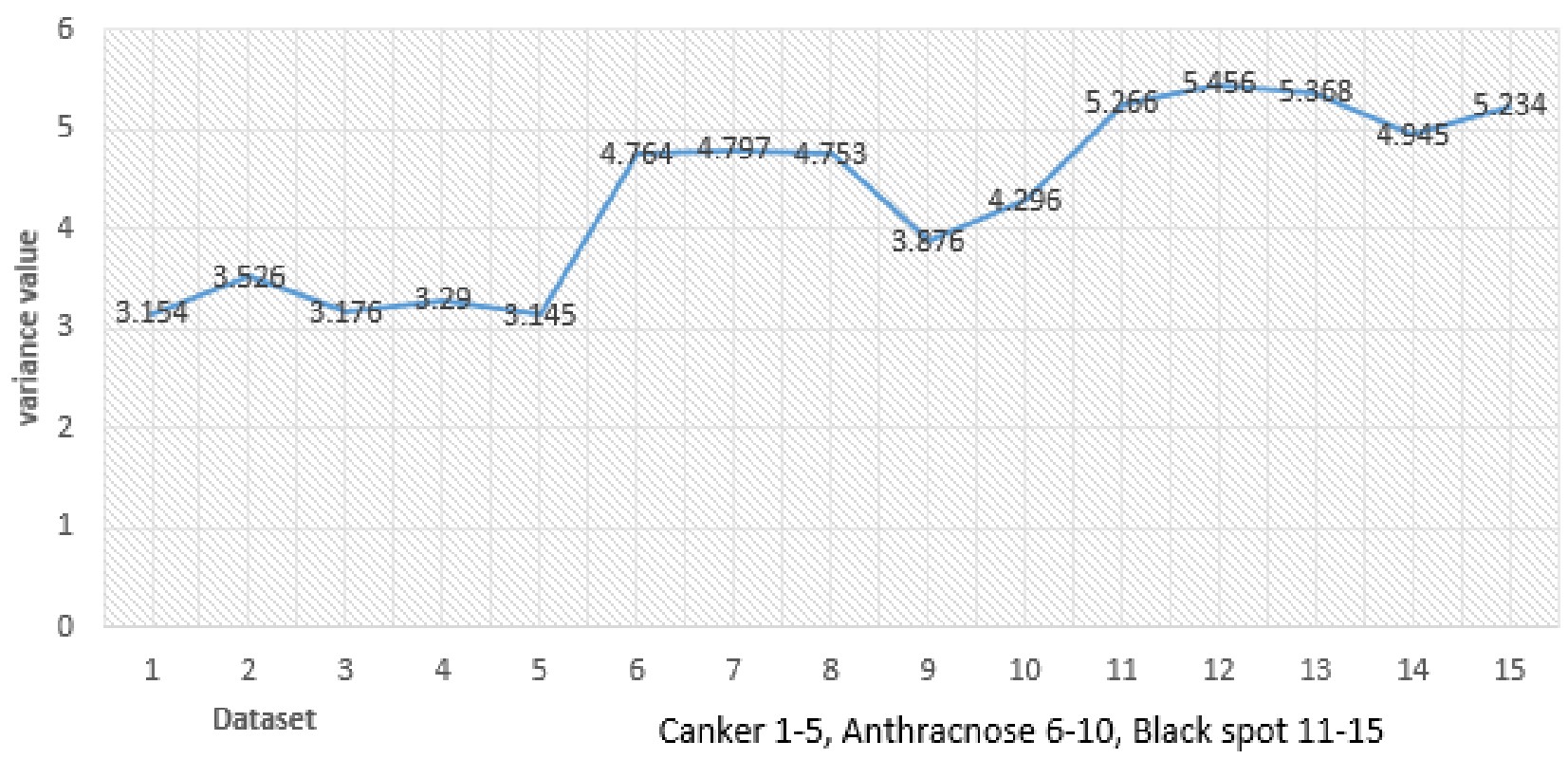

\section{Figure 4. Citrus fruit Variance result graph}

Figure 4. above, reveals variance values plotted against various citrus infections namely canker, anthracnose, and black spot. The graph shows that citrus blackspot disease has the highest curve which implies that our method was able to detect and classify blackspot disease.

\section{Skewness}

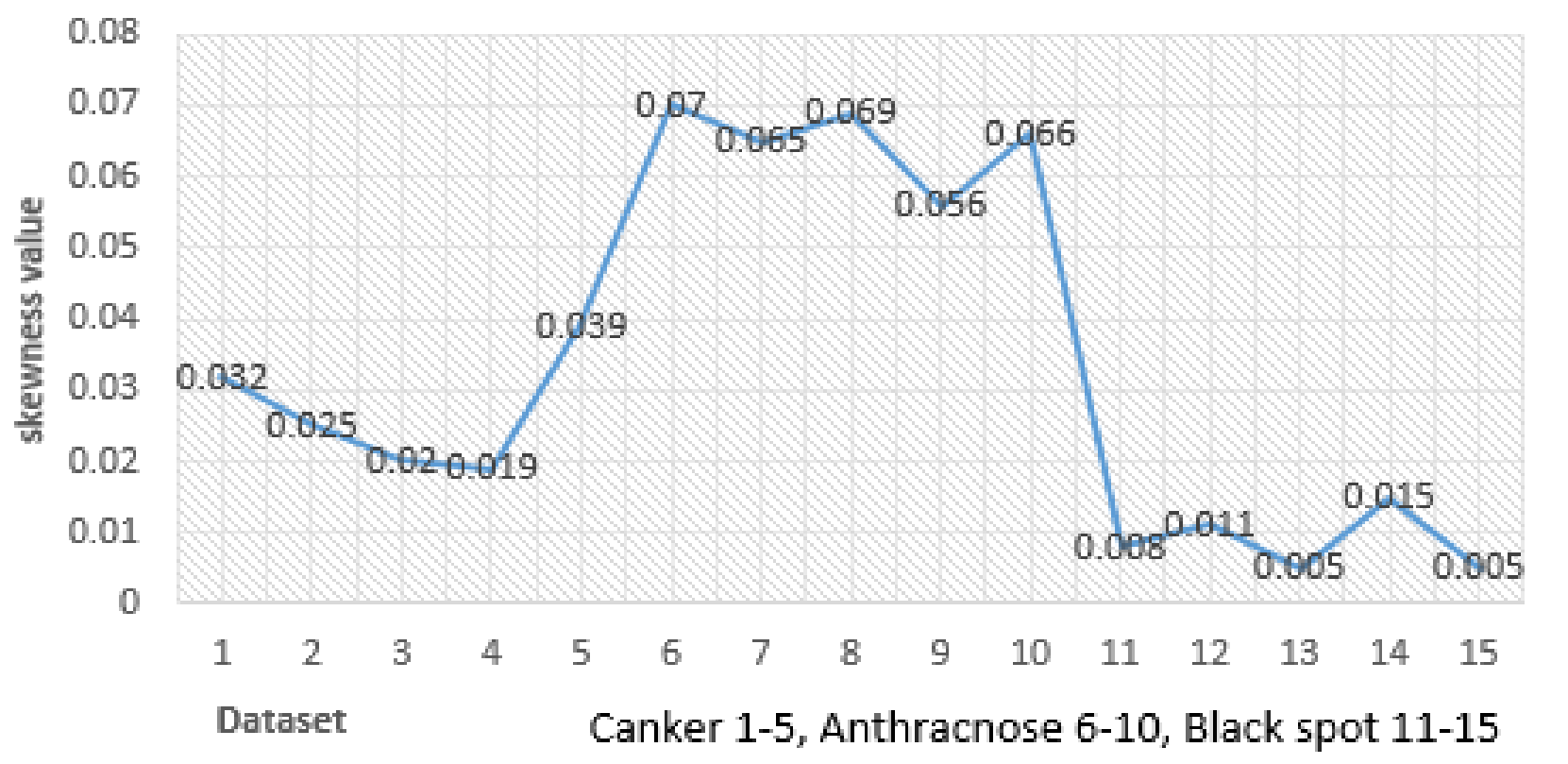

Figure 5. Citrus Fruit Skewness result graph

Figure 5, shows skewness values plotted against various citrus infections namely canker, anthracnose, and black spot. The graph also shows that citrus anthracnose disease has the highest curve which implies that method used in this research work was able to detect and classify anthracnose disease after our computation. 
Table 2. Performance

\begin{tabular}{|c|c|c|c|}
\hline Dataset & Total test sample & Correctly classified & \% Accuracy \\
\hline Anthracnose & 50 & 46 & $96.9 \%$ \\
\hline Black spot & 50 & 50 & $98.7 \%$ \\
\hline Canker & 50 & 48 & $99.1 \%$ \\
\hline Citrus Scab & 50 & $\mathbf{1 9 0}$ & $\mathbf{9 5 \%}$ \\
\hline Combination & $\mathbf{2 0 0}$ & & $97.6 \%$ \\
\hline
\end{tabular}

Table 2, consists of the dataset, total test sample, correctly classified and accuracy value. 50 samples affected with anthracnose disease were used and 46 samples were correctly classified and $96.9 \%$ accuracy recorded. Also, 50 samples affected with black spot disease are used and all samples were correctly classified and $98.7 \%$ accuracy recorded. Moreso, 50 samples affected with canker disease were used and 48 samples are correctly classified and $99.1 \%$ accuracy was recorded. Additionally, 50 samples affected with citrus scab disease were used and 47 samples were correctly classified and $97.6 \%$ accuracy was recorded. In all, the combination of the samples was 200 in total, 190 samples were correctly classified and 95\% accuracy was recorded as the overall performance of the model.

\section{SVM Performance Analysis}

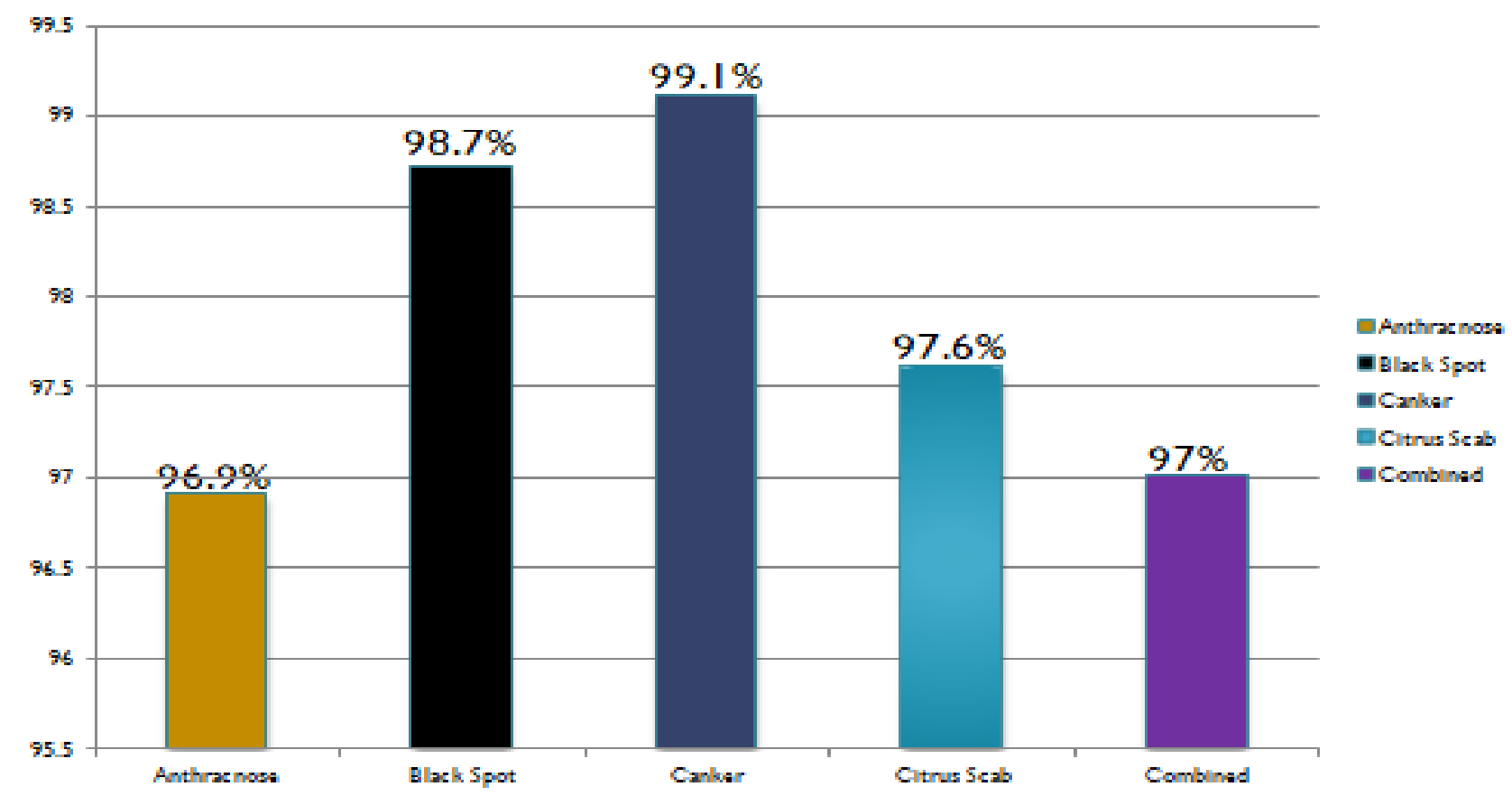

Fig 6. SVM performance analysis

Figure 6 reveals the accuracy value of each disease plotted against various citrus infections namely canker, anthracnose, and black spot. The graph indicates that citrus anthracnose has $96.9 \%$ accuracy, the black spot has $98.7 \%$ accuracy, Canker has $99.1 \%$ accuracy, and citrus scab has $97.6 \%$. All infected and healthy images are tested and overall classification gave 95\% accuracy after the computation. 
Table 3: Model Comparison

\begin{tabular}{|c|c|c|}
\hline Parameters & $\begin{array}{c}\text { Previous Model } \\
\text { (M.I Lali et al) }\end{array}$ & Our Model \\
\hline Classifier & KNN & 200 \\
\hline Total samples & 150 & $\mathbf{9 5 \%}$ \\
\hline Accuracy (\%) & $\mathbf{8 8 . 9 \%}$ & \\
\hline
\end{tabular}

Table 3 shows that the model used in this research work, compared with K-nearest neighbor used by [1], recorded $\mathbf{9 5 \%}$ accuracy with 200 samples used for classification against $\mathbf{8 8 . 9 \%}$ accuracy with 150 samples for classification respectively.

\section{CONCLUSION}

From table 3, it is evidently clear that SVM is a better classifier with $95 \%$ accuracy compared to KNN with $88.9 \%$ accuracy. Thus, model used in this work performs better than the existing model of [1] by $6.1 \%$.

\section{FUTURE WORK}

An automated mobile application can be implemented to help farmers detect various diseases at their early stages.

\section{REFERENCES}

[1] [Ali, H., Lali, M. I., Nawaz, M. Z., Sharif, M., \& Saleem, B. A. (2017). Symptom-based automated detection of citrus diseases using the color histogram and textural descriptors. Computers and Electronics in Agriculture, 138 , 92-104. https://doi.org/10.1016/j.compag.2017.04.008

[2] Hamuda, E., Ginley, B. M., Glavin, M., \& Jones, E. (2018). Improved image processing-based crop detection using Kalman filtering and the Hungarian algorithm. Computers and Electronics in Agriculture, 148(February), 37-44. https://doi.org/10.1016/j.compag.2018.02.027

[3] García-García, I, Taboada-Rodríguez, A, López-Gomez, A and Marín-Iniesta, F. 2013. Active Packaging of Cardboard to Extend the Shelf Life of Tomatoes. Food and Bioprocess Technology 6 (3): 754-761

[4] Sankaran, S., Mishra, A., Ehsani, R. and Davis, C. (2010) A Review of Advanced Techniques for Detecting Plant Diseases. Computers and Electronics in Agriculture, 72, 1-13.

[5] López-García F, Andreu-García G, Blasco J, Aleixos N, Valiente JM (2010) Automatic detection of skin defects in citrus fruits using a multivariate image analysis approach. Comput Electron Agric 71(2):189-197.

[6] Pydipati R, Burks TF, Lee WS (2005) Statistical and neural network classifiers for citrus disease detection using machine vision. Trans ASAE 48(5):2007-2014

[7] Pydipati, R., Burks, T.F., Lee, W.S., 2006. Identification of citrus disease using color texture features and discriminant analysis. Comput. Electron. Agric. 52, 4959. http://dx.doi.org/10.1016/j.compag.2006.01.004

[8] Sanyal P, Patel SC (2008) Pattern recognition method to detect two diseases in rice plants. Imaging Sci J 56(6):7
[9] Sanyal P, Bhattacharya U, Parui SK, Bandyopadhyay SK, Patel S (2007) Color texture analysis of rice leaves diagnosing deficiency in the balance of mineral levels towards improvement of crop productivity. In: 10th International Conference on Information Technology (ICIT 2007). IEEE, Orissa, pp 85-90

[10] Yao Q, Guan Z, Zhou Y, Tang J, Hu Y, Yang B (2009) Application of support vector machine for detecting rice diseases using shape and color texture features. In: 2009 international conference on engineering computation. IEEE, Hong Kong, pp 79-83

[11] Jian Z, Wei Z (2010) Support vector machine for recognition of cucumber leaf diseases. In: 2010 2nd international conference on advanced computer control. IEEE, Shenyang, pp 264-266

[12] Youwen T, Tianlai L, Yan N (2008) The recognition of cucumber disease based on image processing and support vector machine. In: 2008 congress on image and signal processing. IEEE, Sanya, pp 262-267

[13] Huang KY (2007) Application of artificial neural network for detecting Phalaenopsis seedling diseases using color and texture features. Comput Electron Agric $57: 3-11$

[14] Haralick RM, Shanmugam K, Dinstein I (1973) Textural features for image classification. IEEE Trans Syst Man Cybern SMC-3 3:610-62

[15] Hetzroni A, Miles GE, Engel BA, Hammer PA, Latin RX (1994) Machine vision monitoring of plant health. Adv Space Res 14(11):203-212

[16] Jednipat Moonrinta, Supawadee Chaivivatrakul, Matthew N. Dailey, and Mongkol Ekpanyapong (2010) Fruit Detection, Tracking, and 3D Reconstruction for Crop Mapping and Yield Estimation

[17] Mohammed Yesuf (2013) Pseudocercospora leaf and fruit spot disease of citrus: Achievements and challenges in the citrus industry: A review. Vol.4, No.7, 324-328 (2013) http://dx.doi.org/10.4236/as.2013.47046

[18] Meunkaewjinda. A, P.Kumsawat, K.Attakitmongcol and A.Sirikaew.2008 Grape leaf diseases from color imaginary using Hybrid intelligent system", Proceedings of ECTICON.

[19] Nivedit.R. Kakade, Dnyaneswar.D.Ahire (2015) "A Review of Grape Plant Disease Detection" International Research Journal of Engineering and Technology, Volume: 02 Issue: 05 | Aug-2015

[20] Pujari, J.D., Yakkundimath, R., 2013. Grading and classification of anthracnose fungal disease of fruits 
based on statistical texture features. Int. J. Adv. Sci. Technol. 52, 121-132.

[21] Pujari, J.D., Yakkundimath, R., Byadgi, A.S., 2015. Image processing-based detection of fungal diseases in plants. Procedia Comput. Sci. 46, 1802-1808. http://dx.doi. org/10.1016/j.procs.2015.02.137.

[22] Qin, J., Burks, T.F., Ritenour, M.A., Bonn, W.G., 2009. Detection of citrus canker using hyperspectral reflectance imaging with spectral information divergence. J. Food Eng.93,183-191. http://dx.doi.org/10.1016/j.jfoodeng.2009.01.014.

[23] Hocquellet, A., Toorawa, P., Bové, J.M., Garnier, M., 1999. Detection and identification of the two Candidatus Liberobacter species associated with citrus huanglongbing by PCR amplification of ribosomal protein genes of the beta operon. Mol. Cell. Probes 13, 373-379. http://dx.doi.org/10.1006/ mcpr.1999.0263.

[24] Javaid, M.A., Tariq, M.A., Asi, A.A., 2006. Effect of Micronutrients Application on the Yield and Quality of Kinnow Mandarin (Citrus Reticulata Blanco.) 38, 169172.

[25] Almada-Ruiz, E., Martínez-Téllez, M.Á., HernándezÁlamos, M.M., Vallejo, S., Primo- Yúfera, E., Vargas-
Arispuro, I., 2003. Fungicidal potential of methoxylated flavones from citrus for in vitro control of Colletotrichum gloeosporioides, causal agent of anthracnose disease in tropical fruits. Pest Manage. Sci. 59, 1245-1249. http://dx.doi.org/10.1002/ps.747.

[26] Deng, Z., Huang, S., Xiao, S., Gmitter, F.G., 1997. Development and characterization of SCAR markers linked to the citrus tristeza virus resistance gene from Poncirus trifoliata. Genome 704, 697-704.

[27] Quan-Sen Sun, Sheng-Gen Zeng, Yan Liu, Pheng-Ann Heng, De-Shen Xia; 2005. A new method of feature fusion and its application in image recognition. Volume 38 Issue 12, December, 2005. Pages 2437-2448, doi>10.1016/j.patcog.2004.12.013

[28] Mohammad Haghighat, Mohamed Abdel-Mottaleb, Wadee Alhalabi; 2016. Discriminant Correlation Analysis: Real-Time Feature Level Fusion for Multimodal Biometric Recognition. IEEE Transactions on Information Forensics and Security (Volume: 11, Issue: 9, Sept. 2016 ). Page(s): 1984 - 1996 DOI: 10.1109/TIFS.2016.2569061

[29] M.M. El-gayar, H. Soliman, N. meky; 2016. A comparative study of image low level feature Extraction algorithms. http://dx.doi.org/10.1016/j.eij.2013.06.003 